\title{
Use of artificial dielectric for improvement of printed biconical vibrator matching
}

\author{
A. S. Avdushin ${ }^{1}$, A. V. Ashikhmin ${ }^{1}$, Yu. G. Pasternak ${ }^{2}$, S. M. Fedorov² \\ ${ }^{1}$ JSC "IRCOS", Moscow, Russia \\ ${ }^{2}$ Faculty of radio engineering and electronics, Voronezh State Technical University, Voronezh, Russia
}

Email address:

AvdushinAS@gmail.com (A. S. Avdushin),info@ircos.ru (A. V. Ashikhmin), PasternakYG@mail.ru (Yu. G. Pasternak), zar36@yandex.ru (S. M. Fedorov)

\section{To cite this article:}

A. S. Avdushin, A. V. Ashikhmin, Yu. G. Pasternak, S. M. Fedorov. Use of Artificial Dielectric for Improvement of Printed Biconical Vibrator Matching. American Journal of Electromagnetics and Applications. Vol. 2, No. 6, 2014, pp. 49-52. doi: 10.11648/j.ajea.20140206.11

\begin{abstract}
This article shows that use of artificial dielectric in form of two-dimensional array consisted of small plates can transform electrical input impedance of printed biconical vibrator in ultra-wide bandwidth and improve quality of its matching. It is also found that use of this artificial dielectric eliminates deep dip in radiation pattern of planar biconical vibrator in direction normal of its plane at high frequencys.
\end{abstract}

Keywords: Artificial Dielectric, Printed Antenna

\section{Introduction}

In this paper was studied the possibility and efficiency of input impedance transformation for flat biconical vibrator in ultra-wide frequency band by covering it with electrically small metal plates, deposited on both sides of thin dielectric coating of antenna device. Earlier studies [1-4] have shown that structure consisted of pair nanoscale metal plates can have magnetic properties, even in the optical wavelength range. Specifically, in [1] was shown that dual metal nano rods can have significant characteristics. In [2] was shown that such structures can, under certain conditions, exhibit diamagnetic properties and, which is very interesting, even negative values of refractive index in optical wavelength range. In [3, 4], experimental studies of optical metamaterials with negative refractive index are shown.

\section{Description of Technology}

In this article were studied two kinds of plate - in the form of square frame with side length of $8 \mathrm{~mm}$, and - solid square plate with dimensions substantially smaller than wavelength, Fig. 1. In particular, for coating of small metallic frame, it is assumed that due to time-varying magnetic flux penetrating the frame, it will be induce ring (antisymmetric) currents, which flow causes secondary magnetic field. The expectation was on that formation of several magnetic radiators (frames) may increase proportion of magnetic energy in near field of electric vibrator, in which dominates electric field: $\left|E_{\Sigma}\right| /\left|H_{\Sigma}\right| \gg Z_{0}=\sqrt{\mu_{a} / \varepsilon_{a}}$. Alignment balance between electric and magnetic energy in near field of vibrator should improve antenna matching in wide bandwidth.

Advantages of proposed technology are - use technology of multilayer printed circuit boards for antenna production (i.e. high manufacturability), and - the possibility of realizing the function of ultrawideband transformation of antenna input impedance with value of $150 \mathrm{ohms}$ to standard $50 \mathrm{ohms}$ without using a transformer, like tapering transmission line. The disadvantage is - need to use at least two-layer printed circuit board for implementing a metamaterial antenna coating.

In addition, presence of magnetic field component oriented parallel to plane of the frames and vibrator should also induce antisymmetric currents $\vec{J}_{1}$ and $\vec{J}_{2}$ within opposing frames and between vibrator shoulders and frames, because normal vectors $\overrightarrow{n_{1}}$ and $\overrightarrow{n_{2}}$ to opposing sides of metal plates are oriented in opposite directions. Displacement currents in space between plates close antisymmetric currents, which also lead to occurrence of secondary magnetic field.

Also, under influence of vibrator electric field in artificial dielectric plates induced cophased (symmetric in left and right halves of the frame) currents. In impedance is introduced capacitive component due to capacitance between the plates, 
as well as - between plates and vibrator shoulders which leads to substantial decrease of wave resistance of structure and input impedance of vibrator. The equivalent circuit of one period of artificial dielectric structure may be represented as shown at fig. 2 .
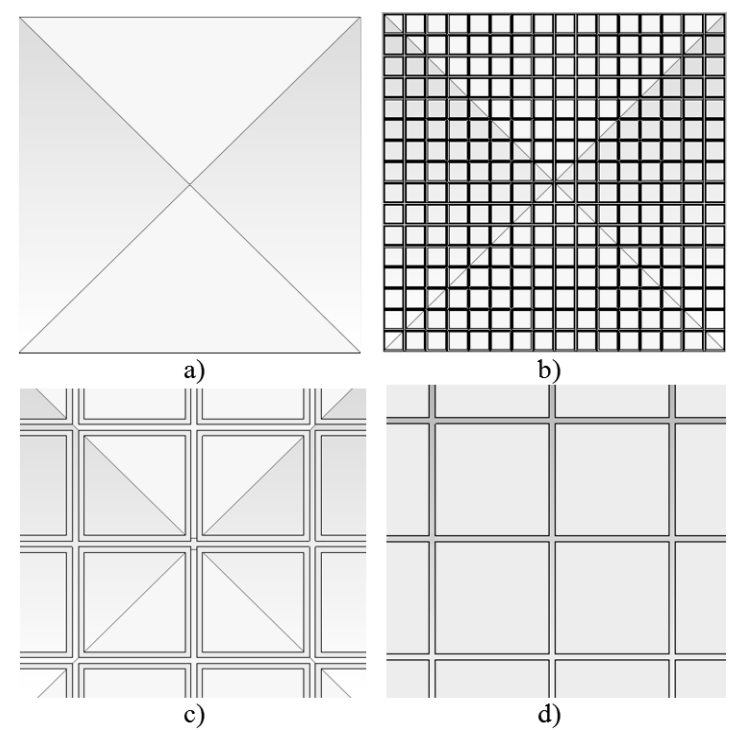

Figure 1. Flat biconical vibrator with side of $160 \mathrm{~mm}$ (a), frame covering (b), geometry of frame (c), geometry of covering solid plates (d). Substrate material - Rogers 4003, thickness - $0.508 \mathrm{~mm}$.

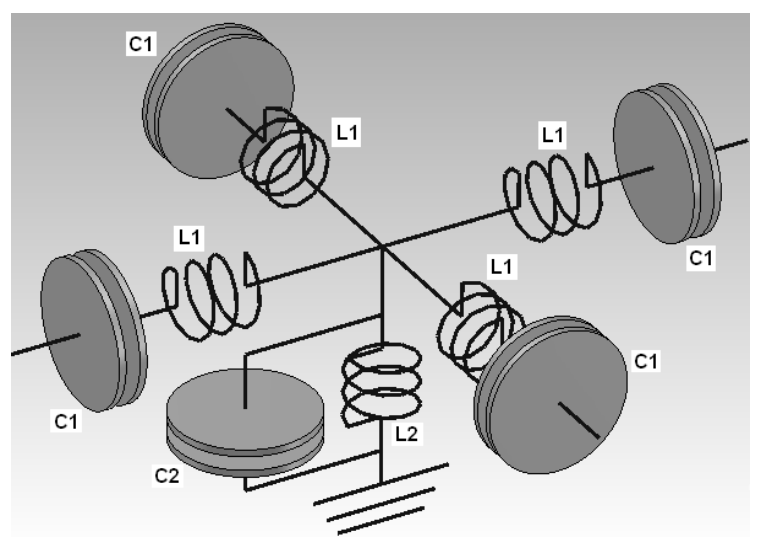

Figure 2. The equivalent circuit of one period of artificial dielectric structure.

Following abbreviations are used: $C 1$ - capacitances, which taking into account the link between plates arranged in adjacent Floquet cells of structure; $L 1$ - inductances of plates (or frames); C2 - capacitance between plates in same cell, and - between plates (or frames) of artificial dielectric and vibrator shoulders; $L 2$ - inductance caused by antisymmetric currents which are induced in result of time-varying magnetic flux penetrating cell of structure, and - antisymmetric currents induced by magnetic field component in plates (or frames) as well as - in vibrator shoulders.

Considering, that period of artificial dielectric structure $d=10 \mathrm{~mm}$ significantly smaller than wavelength in studied frequency range $f \in[0.5 ; 3] \mathrm{GHz}$ and small inductive reactance value of solid square plates, in low-frequency range will be dominate values of distributed impedance determined by reactance $C 1, C 2$ and $L 2$ (because $L 2>L 1$ ). Period of artificial dielectric structure was chosen experimentally to get transformation ratio of $3: 1$. Thus, considering that gap between plates, located in neighboring Floquet cells, equal to gap between plates of artificial dielectric structure and shoulders of structure $(0.5 \mathrm{~mm}$ in this case $)$, the capacitance values between plate and vibrator shoulder is greater than capacitance between plates, located in neighboring Floquet cells: $C 2>C 1$.

At frequencies below resonant frequency of parallel oscillatory circuit with elements $(C 2, L 2)$ considered equivalent circuit corresponds to high-pass filter. In [5-11] studied dispersion characteristics of metamaterials with different structures. In particular, it is shown that in long-wave region, where equivalent circuit of studied artificial dielectric is high pass filter, effective refractive index of material may be negative in wide frequency band.

At higher frequencies (higher than the resonant frequency of series oscillatory circuit with elements $(C 1, L 1)$ and parallel oscillating circuit with elements $(C 2, L 2))$ an equivalent circuit of this structure is low-pass filter that, according to the results of studies [5-11], corresponds to positive values of refractive index of artificial dielectric.

Due to significant shunt capacitance effects of $C 2$ in studied variants of artificial dielectric structure, average input impedance of plane biconical vibrator is significantly reduced: from $150 \mathrm{ohms}$ to about $50 \mathrm{ohms}$, Fig. 3, which is very convenient to match the antenna to feed line with standard impedance of $50 \mathrm{ohms}$. All studied characteristics obtained by using CST Microwave Studio 2012. Fig. 3 shows, that when coating made in form of closed frames the antenna input impedance at high frequencies becomes almost purely active, while coating in form of solid square plates - gives us capacitive component of input impedance at high frequencies.

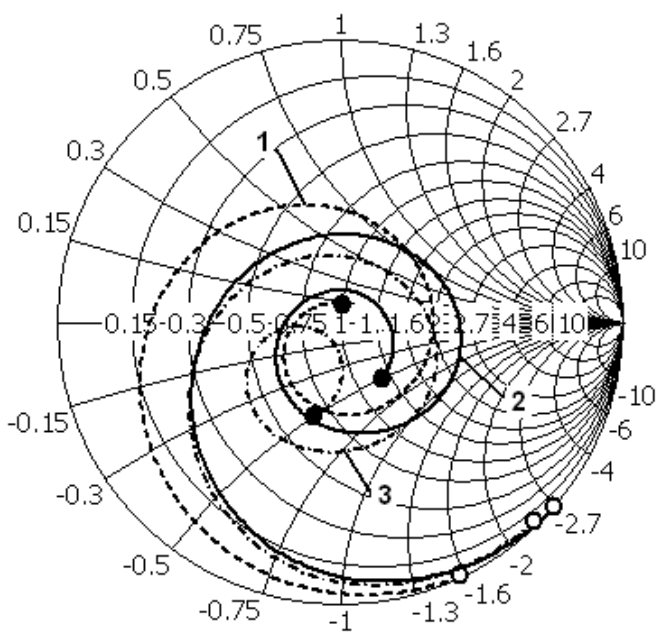

Figure 3. 1) - Vibrator in dielectric layer and uncovered (150 ohms) 2) covering in form of frames (54 Ohm) 3) - covering in form of square plates (54 ohms).

Fig. 4 shows that use of artificial dielectric in form of flat biconical vibrator covering can compensate deep dip in radiation pattern in direction of normal to vibrator plane at 
high frequency region (from 2.5 to $3 \mathrm{GHz}$ ). Cause of deep dip of vibrator without metamaterial covering is significant difference from common-mode current distribution in his shoulders. In vibrator covered with metamaterial at frequency range, where absolute value of refractive index $|n|$ is less than

1 , phase velocity of currents, flowing through vibrator shoulders, substantially increases $v=v_{0} / n$, whereby current distribution tends to common-mode, thus deep dip in radiation pattern in direction of normal to vibrator plane is compensated. Studies have shown that this effect occurs both in case of solid covering plates and frame covering.

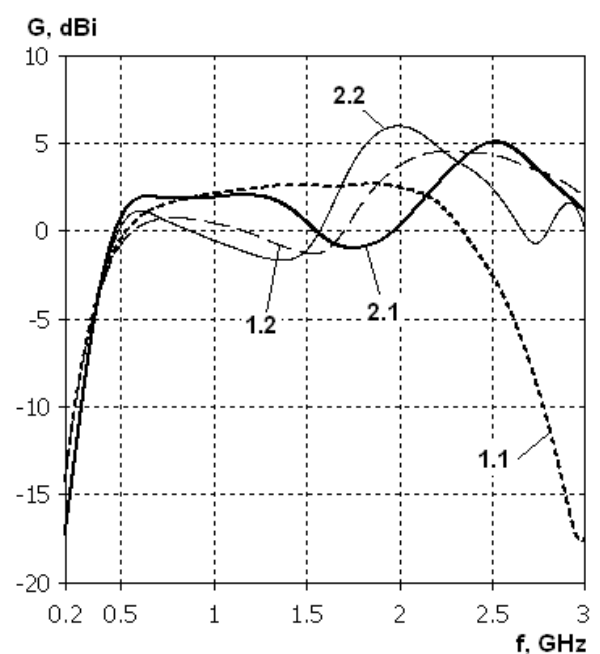

Figure 4. 1.1 - Gain of vibrator in dielectric layer and uncovered in direction of normal to antenna plane; 1.2 - gain of vibrator in dielectric layer and uncovered in direction perpendicular to axis of the antenna; 2.1 - gain of vibrator in dielectric layer and covered by frames in direction of normal to antenna plane; 2.2 - gain of vibrator in dielectric layer and covered by frames in direction perpendicular to axis of the antenna.

Numerical simulation of flat biconical vibrator with covering of researched artificial dielectric and powered by a coplanar line was performed, Fig. 5.

Smith chart for researched antenna in frequency band from 0.5 to $3 \mathrm{GHz}$ is shown at Fig. 6, frequency dependence of VSWR - Fig. 7. Frequency dependences of gain in direction of normal to plane and perpendicular to axis of the antenna are shown at Fig. 8.

Calculations showed that efficiency decrease of antenna including losses in transformer at researched frequency range from 0.5 to $3 \mathrm{GHz}$ is less than $0.35 \mathrm{~dB}$.
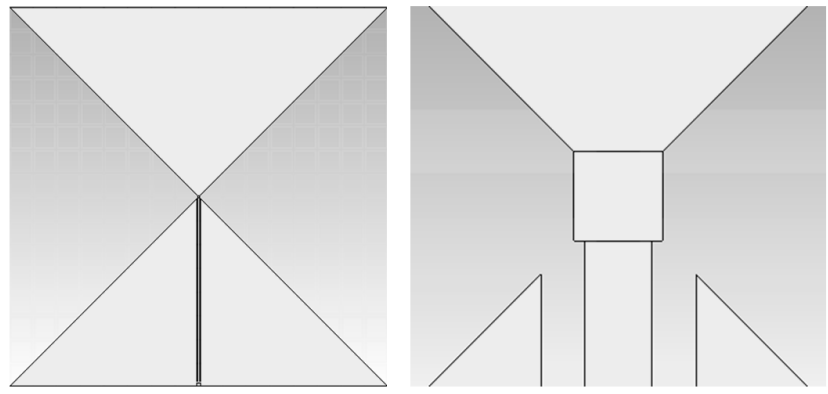

Figure 5. Powering of flat biconical vibrator by coplanar line.

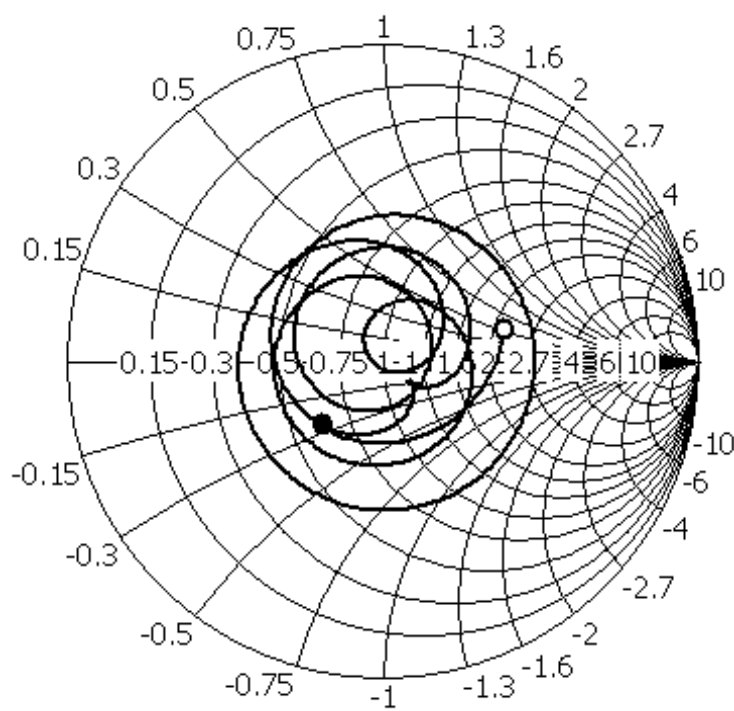

Figure 6. Smith chart at frequency band from 0.5 to $3 \mathrm{GHz}$, transmission line impedance - 50 ohms.

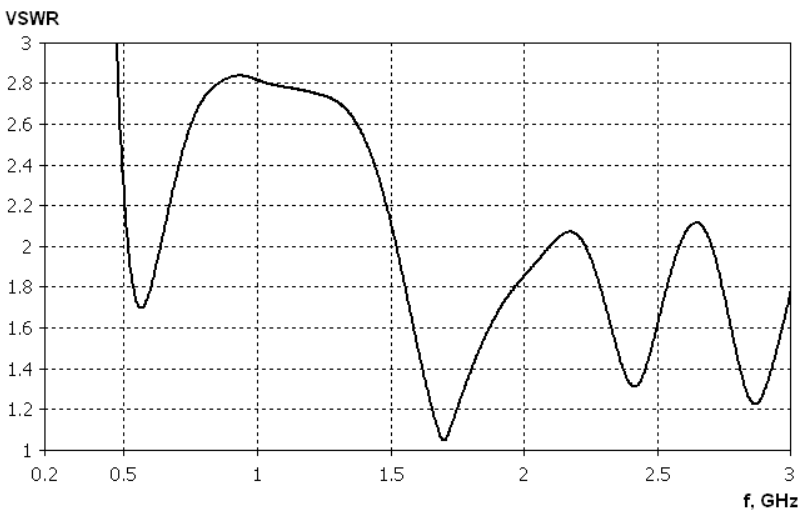

Figure 7. Frequency dependence of VSWR at antenna port, with feed line impedance 50 ohms.

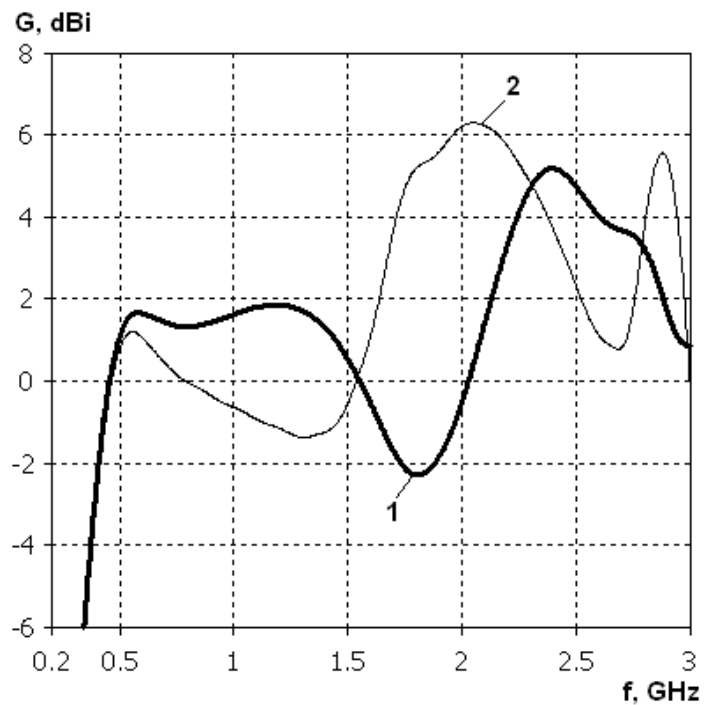

Figure 8. Frequency dependence of antenna gain: 1 - gain in direction of normal to antenna plane, 2 - gain in direction perpendicular to axis of the antenna 


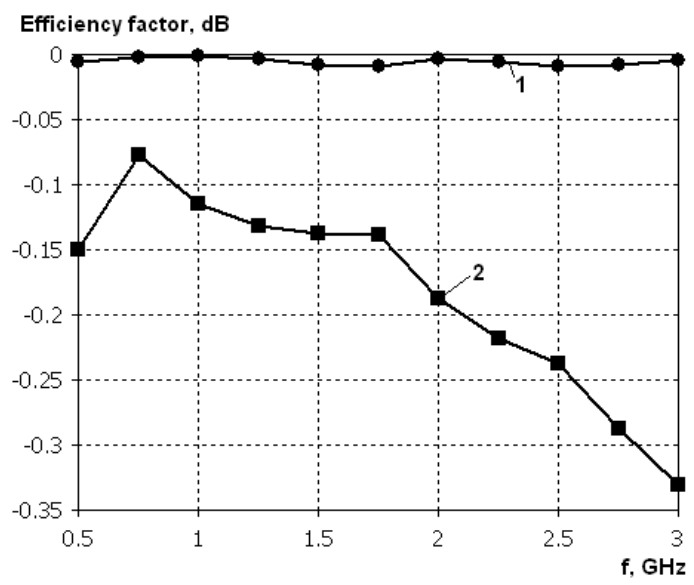

Figure 9. Frequency dependence of antenna efficiency: 1 - efficiency of covered vibrator with powering in its center by external voltage source; 2 antenna efficiency, with included losses in transformer

\section{Conclusion}

Based on results of research, was found that use of artificial dielectric in form of two-dimensional array of plates with small electrical sizes (solid square-shaped plates and square frames) allows you to transform input impedance of printed biconical vibrator in ultrawide bandwidth and improve quality of its matching.

Also found that use of this artificial dielectric eliminates deep dip in radiation pattern of planar biconical vibrator in direction of normal to its plane at high-frequency of researched range.

\section{References}

[1] Lagarkov A.N., Sarychev A.K. (1996) Electromagnetic properties of composites containing elongated conducting inclusions. Phys. Rev. B 53:6318-6336.
[2] Podolskiy V.A., Sarychev A.K., Shalaev V.M. (2002) Plasmon modes in metal nanowires and lefthanded materials. J. Nonlinear Opt. Phys. Mater. 11:65-74.

[3] Shalaev V.M., Cai W.S., Chettiar U.K., Yuan H.K., Sarychev A.K., Drachev V.P., Kildishev A.V. (2005) Negative index of refraction in optical metamaterials. Opt. Lett. 30:3356-3358.

[4] W. Cai, V. Shalaev. Optical Metamaterials. Fundamentals and Applications. Springer. 2010. 200 pp.

[5] W. Cai, V. Shalaev. Optical Metamaterials. Fundamentals and Applications. Springer. 2010. 200 pp.

[6] C. Caloz and T. Itoh. Application of the transmission line theory of left-handed (LH) materials to the realization of a microstrip LH transmission line. Proc. IEEE-AP-S USNC/URSI National Radio Science Meeting, vol. 2, pp. 412-415, 2002.

[7] A. A. Oliner. A periodic-structure negative-refractive-index medium without resonant elements. In URSI Dig., IEEE-AP-S USNC/URSI National Radio Science Meeting 2002, p. 41, San Antonio, June 2002.

[8] L. Liu, C. Caloz, C. Chang, T. Itoh. Forward coupling phenomenon between artificial left-handed transmission lines. J. Appl. Phys., 92, 9 , pp. 5560-5565, 2002.

[9] G. V. Eleftheriades, A. K. Iyer. Planar Negative Refractive Index Media Using Periodically L-C Loaded Transmission Lines. IEEE Trans. Microw. Theory Techniques, 50, 12, pp. 2702-2712, 2002.

[10] A. Lai, C. Caloz, T. Itoh. Composite Right/Left-Handed Transmission Line Metamaterials. IEEE Microwave Magaz. pp. 34-50, 2004.

[11] C. Caloz, T. Itoh. Metamaterials for High-Frequency Electronics. Proc. IEEE, 93, 10, pp. 1744-1752, Oct 2005. 